\title{
Does a Generic Connection Depend Continuously on its Curvature?
}

\author{
Mark A. Mostow ${ }^{1}$ and Steven Shnider ${ }^{2, \star}$ \\ 1 Department of Mathematics, North Carolina State University, Raleigh, NC 27650, USA \\ 2 Department of Mathematics, McGill University, 805 Sherbrooke West, Montreal, P.Q., Canada \\ H3 A2K 6
}

\begin{abstract}
For a principal bundle with semi-simple structure group over a smooth four-dimensional base manifold, the set of connections (gauge potentials) $A$ which are uniquely determined by their curvature (field or field strength) $F$ is generic in the set of all potentials, endowed with the Whitney $C^{\infty}$ topology. However, the operator taking each such field $F$ to its potential $A$ is not continuous. Partial negative results are given concerning the existence of a smaller generic set on which this operator is continuous.
\end{abstract}

\section{Introduction}

$\mathrm{Wu}$ and Yang $[\mathrm{WY}]$ showed that one new aspect of non-abelian gauge theories is the existence of field copies, i.e., gauge inequivalent potentials (connections) with the same gauge field (=field strength =curvature). Since then there has been an interest in describing, either completely or modulo gauge equivalence, the set of gauge potentials with a given gauge field. (See, for example, [C, DX, DD, DW, GY, H1, H2, H3, KC, M, R, So, W].)

The fact that for many fields the potential is unique leads one to another set of questions. What characterizes the set $\mathscr{A}^{*}$ of gauge potentials which are uniquely determined by their curvature? Is $\mathscr{A}^{*}$ generic in the space $\mathscr{A}$ of all connections? What can be said about the mapping $\mathscr{F}$ of function spaces (endowed with the respective Whitney $C^{\infty}$ topologies) taking each potential $A$ to its field $F$ ? Is the inverse of the restriction $\mathscr{F} \mid \mathscr{A}^{*}$ continuous? That is, can one derive estimates for these potentials and their derivatives from similar estimates on the field?

These questions are of interest because they relate to the possibility of doing quantum field theory in the Feynman approach using functional integrals over the space of gauge fields, where the gauge transformations $\varphi$ act tensorially $\left(F \mapsto \varphi^{-1} F \varphi\right)$ as opposed to the space of potentials, where the action is affine and depends on the derivative of the gauge transformation $\left(A \mapsto \varphi^{-1} A \varphi+\varphi^{-1} d \varphi\right)$ [H2]. Other advantages of working on the space of gauge fields instead of gauge potentials are listed in another paper by Halpern [H3].

* Current address: Department of Mathematics, Ben-Gurion University of the Negev, Be'er-Sheva', Israel 
Our main result is:

Proposition. For any gauge field theory on a principal bundle over a fourdimensional base manifold, with semi-simple gauge group, the set $\mathscr{A}^{*}$ of gauge potentials $A$ which are uniquely determined by their field $F$ is generic. In fact, $\mathscr{A}^{*}$ contains an open dense subset of $\mathscr{A}$, the space of all potentials, endowed with the Whitney $C^{\infty}$ topology. However, the operator $\left(\mathscr{F} \mid \mathscr{A}^{*}\right)^{-1}$ taking each such field $F$ to its potential $A$ is not continuous.

We shall prove this result as three theorems, two on genericity and one on discontinuity, which are stated precisely in Sect. 1.

Remark. The first claim (that $\mathscr{A}^{*}$ is generic) has the status of a "folk theorem" among physicists, but to our knowledge, no rigorous proof of it has been published. Other authors (see, for example, DT, DW, H1]) have remarked that at each point of the (4-dimensional) base space, curvature determines connection uniquely in the "generic" case, at least when the gauge group is $\mathrm{SU}(2)$ or SO(3). In contrast, we use the word "generic" in the global sense, which involves the Whitney $C^{\infty}$ topology on the space of all connections (see Sect. 1). Our proof is valid for all semi-simple gauge groups.

The proposition leaves open the following interesting question:

Does there exist a smaller but still generic set $\mathscr{A}^{\prime}$ of connections for which the operator $\left(\mathscr{F} \mid \mathscr{A}^{\prime}\right)^{-1}$ taking $F$ to $A$ is continuous?

In Sect. 1 we discuss some choices of $\mathscr{A}^{\prime}$ which seemed to us likely to work but for which $\left(\mathscr{F} \mid \mathscr{A}^{\prime}\right)^{-1}$ turned out to be discontinuous. Nonetheless, we believe that the answer to the question is yes, and that a suitable set $\mathscr{A}^{\prime}$ can be defined by a transversality condition. We expect to publish results on this problem in the near future.

In the literature one finds several approaches to the problem of characterizing $\mathscr{A}^{*}$, and numerous partial results.

There is a geometric approach using covariant derivatives of curvature, of first and higher orders, together with holonomy groups. This method was used by $\mathrm{Gu}$ and Yang [GY] and by Deser and Drechsler [DD] to study field copy problems, and was developed in detail by the first author of this paper [M]. One drawback of the geometric method is that the covariant derivatives of the curvature cannot be computed, in general, without knowing the connection. Furthermore, solving for the connection involves rank conditions on linear equations which bear no obvious relation to data given about holonomy groups.

A second approach is to work in a particular gauge and to solve for the connection by integration (Halpern [H3] and Weiss [W]). A disadvantage here is that global gauge fixing is not possible in general (Singer [S]).

A third approach, which we shall call the algebraic method, uses the Bianchi identity to solve for the connection from the curvature $F$ and its exterior derivative $d F$ using linear algebra. This technique was used for field copy and other problems by Deser and Teitelboim [DT], Deser and Wilczek [DW], Calvo [C], Halpern [H1], and Roskies [R], among others. It has the advantage of being gauge 
independent and explicit and is the method we adopt in this paper for the genericity and continuity problems.

Doria ([D1, D2]) has attempted to relate the geometric and algebraic approaches and to obtain necessary and sufficient conditions for a curvature to determine its connection uniquely. We have found counterexamples to his results, however [MS].

In Sect. 1 we review the language of bundles and use it to state our results precisely. We prove the theorems on genericity in Sect. 2 and the theorem on discontinuity in Sect. 3. The example used to prove discontinuity is related to an example of a singular potential with non-singular field (in a non-abelian gauge theory), which may be of interest in its own right.

Conventions. We observe the convention of summing over repeated indices. Alternating tensors (defined without an averaging factor) are denoted $\varepsilon_{\mu \nu \sigma \tau}, \varepsilon_{i j k}$, etc. Space-time indices in tensors and connections are denoted by Greek letters, and Lie algebra indices by Roman letters.

\section{Formalism and Precise Statements of Results}

Let $P \rightarrow M$ be a $C^{\infty}$ principal bundle over a connected 4-dimensional base manifold $M$, and let the structure group $G$ (acting on $P$ on the right) be a semi-simple finitedimensional Lie group with Lie algebra $\mathfrak{g}$. (See $[\mathrm{KN}, \mathrm{Gr}]$ for definitions of bundles and related concepts.) Connection 1 -forms on $P$ will be denoted $A$ and their curvature 2-forms $F$. We shall sometimes refer to connections as gauge potentials and to curvatures as gauge fields or field strengths, though strictly speaking a gauge potential, respectively, field, is a locally defined pull-back of a connection, respectively, curvature, to $M$ via some gauge (local section of $P \rightarrow M$ ).

To define the (Whitney) $C^{\infty}$ topology on the space $\mathscr{A}$ of all smooth $\left(C^{\infty}\right)$ connections on $P \rightarrow M$ it is useful, albeit somewhat tedious, to identify $\mathscr{A}$ with $\Gamma E$, the set of all (smooth) sections of a bundle $E \rightarrow M$ whose fiber $E_{x}$ over $x \in M$ is a certain finite-dimensional affine space. Let $P_{x}$ be the fiber of $P$ over $x$. The elements of $E_{x}$ are the $\mathrm{Ad}_{G}$-equivariant sections over $P_{x}$ of the bundle $\operatorname{Hom}(T P, \mathfrak{g}) \rightarrow P$ which restrict on $T\left(P_{x}\right)$ to the standard identification of tangent spaces to $P_{x}$ with $g$ (corresponding to the Maurer-Cartan form on $G \simeq P_{x}$ ). A section of $E \rightarrow M$ is an $\mathrm{Ad}_{G}$-equivariant $\mathfrak{g}$-valued one-form on $P$ which restricts to the Maurer-Cartan form on each fiber $P_{x}$; that is, each section of $E$ is a connection form on $P$. The space of connections $\mathscr{A}=\Gamma E$ is given the Whitney $C^{\infty}$ topology, defined here either by taking the definition of that topology on function spaces $C^{\infty}(X, Y)[\mathrm{GG}$, p. 42] and modifying it in an obvious way for spaces of sections, or, equivalently, by regarding $\Gamma E$ as a subspace of $C^{\infty}(M, E)$.

Remark 1.1. A sequence of functions $f_{i} \in C^{\infty}(X, Y)$ converges to $f$ in the Whitney $C^{\infty}$ topology if and only if for each integer $m \geqq 0$ there exist a compact set $K_{m} \subset X$ and an integer $N_{m}$ such that the $f_{i}$ with $i>N_{m}$ all equal $f$ on $X-K_{m}$, while the sequence $j^{m} f_{i}(x)$ of $m$-jets of $f_{i}$ converges uniformly to $j^{m} f(x)$ on $K_{m}$ [using any metric on the jet manifold $\left.J^{m}(X, Y)\right][\mathrm{GG}$, p. 43].

To relate the affine bundle $E \rightarrow M$ to more familiar vector bundles we first arbitrarily choose a fixed "reference" connection $A^{0}$. For every connection $A$, 
$A-A^{0}$ is an $\mathrm{Ad}_{G}$-equivariant $\mathrm{g}$-valued one-form on $P$ restricting to zero on each fiber $P_{x}$. In a canonical way every such form can be identified with an $(\operatorname{ad} P)$-valued one-form on $M$. (Here ad $P=P \times_{G} g$ is the vector bundle associated to $P$ by the adjoint representation of $G$ on $\mathfrak{g}$.)

[Remark 1.2. Locally one can think of an $(\operatorname{ad} P)$-valued $k$-form on $M$ as an ordinary $\mathfrak{g}$-valued $k$-form on $M$, pulled back from a form of type $\operatorname{ad}_{G}$ on $P$ via a local gauge.]

In other words, by choosing $A_{x}^{0}$ as an origin in the affine space $E_{x}$ we identify $E_{x}$ with the vector space $T_{x}^{*} M \otimes(\operatorname{ad} P)_{x}$, which is the fiber $E_{x}^{1}$ over $x$ of a vector bundle $E^{1}=T^{*} M \otimes(\operatorname{ad} P)$ over $M$. More generally, the $A_{d_{G}}$-equivariant $\mathfrak{g}$-valued $k$-forms on $P$ whose interior products with all vertical vectors are zero correspond canonically with the $(\operatorname{ad} P)$-valued $k$-forms on $M$, that is, with sections of the vector bundle $E^{k}=\left(\Lambda^{k} T^{*} M\right) \otimes$ ad $P \rightarrow M$.

In particular, we define $\mathscr{C}$ to be $\Gamma E^{2}$ with the Whitney $C^{\infty}$ topology. By the curvature operator we mean the mapping $\mathscr{F}: \mathscr{A} \rightarrow \mathscr{C}$, easily seen to be continuous, which takes each connection $A \in \mathscr{A}$ to its curvature $F=d A+\frac{1}{2}[A \wedge A] \in \mathscr{C}$. We remark that $\mathscr{F}$ is not onto; that is, not every 2 -form in $\mathscr{C}$ is a curvature. (This fact is well known, and can be seen easily by studying the jet map $\mathscr{F}_{k}: J^{k+1} E \rightarrow J^{k} E^{2}$, taking $j^{k+1} A$ to $j^{k} F$. For large enough $k$, the domain has smaller dimension than the range, so $\mathscr{F}_{k}$ and hence $\mathscr{F}$ cannot be onto.)

A generic set of connections (or set of generic connections, by abuse of terminology) is defined to be a subset of $\mathscr{A}$ which contains a residual subset of $\mathscr{A}$. We recall [GG, pp. 44] that by definition, a residual subset is a countable intersection of open dense subsets, and that every residual subset of $C^{\infty}(X, Y)$ is dense. The proof of this result can be modified easily for spaces of sections. In particular, every residual subset of $\mathscr{A}$ is dense.

We now formulate in bundle language the algebraic or Bianchi identity method, alluded to in the Introduction, for determining a connection from its curvature under suitable conditions. The Lie bracket on the fibers of ad $P$ induces a wedge-bracket operation $[\cdot \wedge \cdot]$ on the $(\operatorname{ad} P)$-valued differential forms on $M[\mathrm{Gr}$, Vol. II, p. 312]. For any fixed connection $A$ with curvature $F$, the operation $\operatorname{ad}_{F}: B \rightarrow[F \wedge B]$ from $(\operatorname{ad} P)$-valued 1 -forms to 3 -forms on $M$ is induced by a vector bundle $\operatorname{map~ad}_{F}: E^{1} \rightarrow E^{3}$. Since $\operatorname{dim} M=4$, the fibers of both bundles have dimension 4 dimg. By choosing an arbitrary reference connection $A^{0}$ we can write the Bianchi identity $d F=[F \wedge A]$ in the form

$$
d F-\left[F \wedge A^{0}\right]=\left[F \wedge\left(A-A^{0}\right)\right]=\operatorname{ad}_{F}\left(A-A^{0}\right),
$$

or by abuse of notation $d F=\operatorname{ad}_{F}(A)$. On the set $U$ in $M$ where $\operatorname{ad}_{F}$ is invertible, $A$ is uniquely determined by $F$ via

$$
A=A^{0}+\operatorname{ad}_{F}^{-1}\left(\mathrm{dF}-\left[\mathrm{F} \wedge \mathrm{A}^{0}\right]\right),
$$

or by abuse of notation

$$
A=\operatorname{ad}_{F}^{-1}(d F) \text {. }
$$

If $U$ is open and dense in $M$, Eq. (1.1) determines $A$ uniquely on all of $M$, by continuity. 
Let $\mathscr{A}^{* *}$ denote the space of connections $A$ for which $\operatorname{ad}_{F}$ is invertible on an open dense set in $M$. Then $\mathscr{A}^{* *}$ is a subspace of $\mathscr{A}^{*}$, the space of connections uniquely determined by their curvature. (In fact, it is a proper subspace, by an example in [MS].)

Theorem 1. For a principal bundle with real or complex semi-simple structure group and 4-dimensional base manifold, the set $\mathscr{A}^{* *}$, and hence the set $\mathscr{A}^{*}$ of connections uniquely determined by their curvature, is generic.

Remark 1.3. Theorem 1 will follow from Theorem $1^{\prime}$, which states that certain subspaces of $\mathscr{A}^{* *}$ are open and dense in $\mathscr{A}$. This is stronger than genericity.

Formulas (1.1) and (1.2) for $A$ fail where $\mathrm{ad}_{F}$ is not invertible only because they involve division by the determinant of $\mathrm{ad}_{F}$. More precisely, choose a Riemannian or Lorentzian metric on $M$ and define a Hodge star isomorphism * (defined only up to a $\pm \operatorname{sign}$ if $M$ is not oriented) on $(\operatorname{ad} P)$-valued forms on $M$ by using the isomorphism * $\otimes$ id from $\Lambda^{k} T^{*} M \otimes \operatorname{ad} P$ to $\Lambda^{4-k} T^{*} M \otimes \operatorname{ad} P$, where $*$ is the usual Hodge star operator [Fl, p. 15]. Then ${ }^{*} \mathrm{ad}_{F}$ is an endomorphism of $E^{1}$, and the singular set in $M$ where $\mathrm{ad}_{F}^{-1}$ fails to exist is just the zero set of the function $\operatorname{det}\left({ }^{*} \operatorname{ad}_{F}\right): M \rightarrow \mathbb{C}$ (or $\mathbb{R}$ ). [The \pm ambiguity disppears in $\operatorname{det}\left({ }^{*} \mathrm{ad}_{F}\right)$. Changing the metric just multiplies det $* \mathrm{ad}_{F}$ by a smooth nowhere zero function.] Since in local coordinates

$$
\left(* \operatorname{ad}_{F}\right)^{-1}=\frac{\left(\text { matrix of cofactors of } * \mathrm{ad}_{F}\right)^{\text {transpose }}}{\operatorname{det} * \mathrm{ad}_{F}},
$$

we should be able to control the relation of $A$ and $F$ near the singular set by putting conditions on the function det ${ }^{*} \operatorname{ad}_{F}$. (But see Remark 3.2 in Sect. 3 for evidence to the contrary.)

Let $\mathscr{A}_{k}, k=0,1,2, \ldots, \infty$, be the subspace of $\mathscr{A}$ consisting of connections for which det $\operatorname{ad}_{F}$ and its partial derivatives of order $\leqq k$ do not all vanish simultaneously at any point in $M$. In jet notation [GG, p. 37]

$$
\mathscr{A}_{k}=\left\{\left.A \in \mathscr{A}\right|^{k}\left(\operatorname{det}^{*} \operatorname{ad}_{F}\right)(x) \neq 0 \text { for all } x \in M\right\} \text {. }
$$

There are inclusions

$$
\mathscr{A}_{\ddagger} \mathscr{A}^{*} \supsetneqq \mathscr{A}^{* *} \supsetneqq \mathscr{A}_{\infty} \supset \ldots \supset \mathscr{A}_{k+1} \supset \mathscr{A}_{k} \supset \ldots \supset \mathscr{A}_{1} \supset \mathscr{A}_{0} .
$$

Theorem 1'. If $M$ is 4-dimensional, $G$ is semi-simple, and $k \geqq 8 \operatorname{dim} G$, then $\mathscr{A}_{k}$ is generic; in fact, it is an open dense subspace of $\mathscr{A}$.

Remark 1.4. A much lower value of $k$ probably works. On the other hand, if $G=\mathrm{SU}(2)$ or $\mathrm{SO}(3)$, then $\mathscr{A}_{1}$ equals $\mathscr{A}_{0}$, since $\operatorname{det}\left({ }^{*} \mathrm{ad}_{F}\right)$ is then the square of a function of $F$, by the formula of Wu and Yang quoted in [DT], while $\mathscr{A}_{0}$ is not dense, hence not generic, since it contains only connections for which $\operatorname{det}^{*} \operatorname{ad}_{F}$ is always positive on $M$ or always negative.

In light of the continuity result in the Mather Division Theorem [GG, p. 95, especially Note 5] (which concerns division by smooth functions nowhere vanishing to infinite order), one might hope that the restriction of the curvature operator to $\mathscr{A}_{\infty}$ would be continuously invertible. Our next theorem says that this is not the case, however. 
Theorem 2. Let $P$ be any principal bundle over a 4-dimensional base manifold $M$ with gauge group $G=\mathrm{SU}(2)$ or $\mathrm{SO}(3)$. The curvature operator $\mathscr{F}$, restricted to $\mathscr{A}^{*}$ or even to $\mathscr{A}_{\infty}$, does not have a continuous inverse.

Question. Is there some other generic subspace $\mathscr{A}^{\prime} \subset \mathscr{A}$ for which $\left(\mathscr{F} \mid \mathscr{A}^{\prime}\right)^{-1}$ is continuous?

Our proof of Theorem 2 actually shows that $\left(\mathscr{F} \mid \mathscr{A}_{28}\right)^{-1}$ is not continuous under the given hypotheses. Possibly $\left(\mathscr{F} \mid \mathscr{A}_{k}\right)^{-1}$ is continuous for some generic $\mathscr{A}_{k}$ with $k<28$, but we are doubtful, especially in light of Remark 3.2 of Sect. 3 .

It seems to us more fruitful to examine subspaces $\mathscr{A}^{\prime}$ defined by other types of conditions. In particular, we have conjectured that the set $\mathscr{A}^{\prime}$ of connections $A$ whose curvatures $F$ are transversal to the zero set of the polynomial $F \mapsto \operatorname{det} * \operatorname{ad}_{F}$ (more exactly, to every stratum of a certain Whitney stratification of the zero set) satisfies the conditions on $\mathscr{A}^{\prime}$ in the question. We have sketched a proof, which we hope to complete and publish soon.

\section{Proof of Theorem $1^{\prime}$ (Genericity of $\mathscr{A}_{k}$ )}

The proof that $\mathscr{A}_{k}$ is an open dense subset of $\mathscr{A}$ will be an application of transversality theory [GG], together with a claculation showing that $\operatorname{det} * \mathrm{ad}_{F}$ need not always be zero if $G$ is semi-simple.

Definition. Let $J$ be a smooth finite dimensional manifold and $S$ a subset of $J$. We call $S$ a locally algebraic subset of $J$ if there are $C^{\infty}$ local coordinate charts covering $J$ in which $S$ is (locally) an algebraic set (zero set of a collection of polynomials in the local coordinates).

A standard result on algebraic sets [Mil, p. 15] implies that a locally algebraic subset is a locally finite (and hence countable) union of connected smooth (actually locally analytic) submanifolds of $J$. It is clearly closed.

Definition. The codimension of a locally algebraic subset $S \subset J$ is the smallest codimension (in $J$ ) of all submanifolds in one, and hence any, locally finite union of submanifolds whose union is $S$.

Let $E \rightarrow M$ be the bundle defined in Sect. 1 and let $J^{k+1}(E)$ be the manifold of $(k+1)$-jets $j^{k+1} A(x)$ [GG, p. 37] of its sections, that is, of connections $A$. Let $J^{k}(M, \mathbb{C})$ be the manifold of $k$-jets of functions $f: M \rightarrow \mathbb{C}$, and let $\sigma_{k}: J^{k+1}(E) \rightarrow J^{k}(M, \mathbb{C})$ be defined by

$$
\sigma_{k}:\left(j^{k+1} A\right)(x) \mapsto j^{k}\left(\operatorname{det}^{*} \operatorname{ad}_{F}\right)(x),
$$

where $F$ is the curvature of $A$. Choose local coordinates

$$
\left(x^{\mu}, A_{\mu}^{i}, \partial_{v_{1}} A_{\mu}^{i}, \partial_{v_{1}} \partial_{v_{2}} A_{\mu}^{i}, \ldots, \partial_{v_{1}} \ldots \partial_{v_{k+1}} A_{\mu}^{i}\right) \quad \text { on } \quad J^{k+1}(E) ;
$$

here $x=\left(x^{\mu}\right)$ are 4 local coordinates on $M, A_{\mu}^{i}$ are the components of $A$ in some local gauge ( $\mu$ is a space-time index, $i$ a Lie algebra index), and $\partial_{v_{1}} \ldots \partial_{v_{s}} A_{\mu}^{i}$ is an $s^{\text {th }}$ partial space-time derivative of $A_{\mu}^{i}$ at $x$. In this chart $\sigma_{k}$ is a polynomial in the 
coordinates, independent of the $x^{\mu}$. Hence

$$
S_{k}=(\operatorname{def})\left\{\left(j^{k+1} A\right)(x) \in J^{k+1}(E) \mid \sigma_{k}\left(\left(j^{k+1} A\right)(x)\right)=0\right\}
$$

is a locally algebraic subset of $J^{k+1} E$.

Observe that $\mathscr{A}_{k}$ can be viewed as the set of all $A$ for which the $(k+1)$-jet section $j^{k+1} A: M \rightarrow J^{k+1} E$ has an image which misses $S_{k}$. The proof of Theorem $1^{\prime}$ thus reduces to the following two lemmas.

Lemma 1. If $\operatorname{dim} M=4, G$ is semi-simple, and $k \geqq 8 \operatorname{dim} G$, then $\operatorname{codim} S_{k} \geqq 5$. Hence $j^{k+1} A$ is transversal to each submanifold in any locally finite collection whose union is $S_{k}$ if and only if the image of $j^{k+1} A$ misses $S_{k}$ (by [GG, p. 51]).

Lemma 2. Let $B \rightarrow M$ be any smooth fiber bundle. If $W_{1}, W_{2}, \ldots$ are countably many submanifolds of $J^{r}(B)$, each of codimension $>\operatorname{dim} M$, and if $S=\bigcup_{i} W_{i} \subset J^{r} B$ is closed, then the subset $Z \subset \Gamma B$ consisting of the sections $f: M \rightarrow B$ for which $j^{r} f$ misses $S$ is open and dense in the Whitney $C^{\infty}$ topology.

The proof of Lemma 1 depends in turn upon an algebraic result:

Lemma 3. There exists a curvature $F$ for which $\operatorname{det}^{*} \mathrm{ad}_{F}$ is not identically zero, provided that $\operatorname{dim} M=4$ and $G$ is semi-simple.

Remark 2.1. The function $\operatorname{det} * \mathrm{ad}_{F}$ is defined only if $\operatorname{dim} M=4$.

Remark 2.2. If the commutator subalgebra of $\mathfrak{g}$ does not equal $\mathfrak{g}$, then $[F \wedge B]$ is always a 3 -form with values in the proper subalgebra $[\mathfrak{g}, \mathfrak{g}]$. Hence $\mathrm{ad}_{F}$ cannot be isomorphism and det $* \operatorname{ad}_{F} \equiv 0$. We did not study the case where $[\mathfrak{g}, \mathfrak{g}]=\mathfrak{g}$ but $\mathfrak{g}$ is not semi-simple.

Remark 2.3. In the case $G=\mathrm{SU}(2)$ or $\mathrm{SO}(3)$, Lemma 3 follows from the explicit formulas of Deser and Teitelboim [DT] or of Wu and Yang [DT, footnote 6].

Proof of Lemma 1. The function $\sigma_{1}\left(j^{1} A\right)=\operatorname{det}^{*} \mathrm{ad}_{F}$ is a homogeneous polynomial of $\operatorname{degree} 4 \operatorname{dim} G$ in the quantities

$$
F_{\mu \nu}^{j}=\partial_{\mu} A_{v}^{j}-\partial_{v} A_{\mu}^{j}+c_{i l}^{j} A_{\mu}^{i} A_{v}^{l} .
$$

We can decompose it as $\sigma_{1}=f\left(\partial_{\mu} A_{v}^{i}\right)+g\left(A_{v}^{i}, \partial_{\mu} A_{v}^{i}\right)$, where $f$ is a homogeneous polynomial of degree $4 \operatorname{dim} G$, and $g$ is a sum of monomials of degree at least 2 in the $A_{v}^{i}$ variables and at most $4 \operatorname{dim} G-1$ in the $\partial_{\mu} A_{v}^{i}$ variables. Furthermore, $f$ is not identically zero, since if (using Lemma 3) $F$ is a curvature for which $\left(\operatorname{det} * \operatorname{ad}_{F}\right)\left(x_{0}\right) \neq 0$, then

$$
A_{\mu}(x)=\frac{1}{2} F_{\tau \mu}\left(x_{0}\right)\left(x^{\tau}-x_{0}^{\tau}\right)
$$

is a connection vanishing at $x_{0}$ whose curvature equals $F_{\tau \mu}\left(x_{0}\right)$ at $x_{0}$, so that

$$
0 \neq\left(\operatorname{det} * \operatorname{ad}_{F}\right)\left(x_{0}\right)=\sigma_{1}\left(j^{1} A\right)\left(x_{0}\right)=f\left(\partial_{\mu} A_{v}^{i}\right)
$$

Now let $k=8 \operatorname{dim} G$ (the case $k>8 \operatorname{dim} G$ will follow from this case since $\operatorname{codim} S_{k}$ is non-decreasing in $k$ ). Consider the map $h_{\varrho}$ from a coordinate neighborhood in $J^{k+1} E$ to $\mathbb{C}$ defined by $h_{\varrho}:\left(j^{k+1} A\right)(x) \rightarrow\left(\partial_{\varrho}^{k}\left(\operatorname{det}^{*} \operatorname{ad}_{F}\right)\right)(x)$ for fixed 
space-time index $\varrho$. In this polynomial the sum of monomials of highest degree in the coordinates $\partial_{\varrho}^{2} \partial_{\mu} A_{v}^{i}$ (namely, degree $\left.k / 2\right)$ is easily computed to be $k ! f\left(\partial_{\varrho}^{2} \partial_{\mu} A_{v}^{i}\right)$, which by previous calculation is not identically zero. Furthermore, $h_{\varrho}$ does not involve the variables $\partial_{\tau}^{2} \partial_{\mu} A_{v}^{i}$ for $\tau \neq \varrho$. These two facts, together with results of Whitney on algebraic sets [Mil, pp. 10-15], an inductive argument, and the Frobenius criterion for integrability of a distribution (span of vector fields) $[\mathrm{KN}$, p. 10] imply the following: $h_{\varrho}^{-1}(0)$ can be decomposed into a locally finite union of connected submanifolds, each having at every point a normal vector (relative to the standard Euclidean metric in the given local coordinate system) with a nonzero component in some $\left(\partial_{\rho}^{2} \partial_{\mu} A_{v}^{i}\right)$ direction but no component in any $\left(\partial_{\tau}^{2} \partial_{\mu} A_{v}^{i}\right)$ direction (see Appendix 1 for details). Also, the composition $\sigma_{1} \circ \pi$ taking $\left(j^{k+1} A\right)(x)$ to $\left(\operatorname{det}^{*} \operatorname{ad}_{F}\right)(x)$ is independent of the variables $\partial_{\varrho}^{2} \partial_{\mu} A_{v}^{i}$ for all $\varrho$. It follows that the intersection of the zero sets of $\sigma_{1}^{\circ} \pi$ and the four $h_{\varrho}$ 's is of codimension $\geqq 5$; moreover, it contains $S_{k}$, locally. QED

Proof of Lemma 2. By the Thom Transversality Theorem ([GG, p. 54], adapted to sections of a bundle instead of functions), $Z$ is residual in $\Gamma J$ and hence dense. The openness of $Z$ can easily be proved directly. Let $d$ be a metric on $J^{r}(B)$ and let $f \in Z$. Since $S \subset J^{r}(B)$ is closed and $j^{r} f$ misses $S$, there is a continuous function $\delta: M \rightarrow \mathbb{R}^{+}$ with $d\left(j^{r} f(x), S\right) \geqq \delta(x)$ for all $x$. But then

$$
\left\{g \in \Gamma B \mid d\left(j^{r} f(x), j^{r} g(x)\right)<\delta(x) \text { for all } x\right\}
$$

is an open neighborhood of $f$ contained in $Z$ in the Whitney $C^{r}$, and hence $C^{\infty}$, topology (see [GG, p. 43]).

Proof of Lemma 3. As observed by Deser and Teitelboim [DT], for any point $x_{0} \in M$ and any $(\operatorname{ad} P)$-valued 2 -form $F^{\prime}$ on $M$ there exists a connection $A$, given locally by $A_{\mu}(x)=\frac{1}{2} F_{\tau \mu}\left(x_{0}\right)\left(x^{\tau}-x_{0}^{\tau}\right)$, say, whose curvature $F$ coincides with $F^{\prime}$ at $x_{0}$. Hence it suffices to find any set of components $F_{\mu \nu}=F_{\mu \nu}\left(x_{0}\right)$ which satisfy $\left(\operatorname{det} * \operatorname{ad}_{F}\right)\left(x_{0}\right) \neq 0$.

Since complexifying $\mathfrak{g}$ does not change the problem, we may assume that $\mathfrak{g}$ is a complex semi-simple Lie algebra (see [BK] for Lie algebra definitions and results). Choose a basis of $\mathfrak{g}$ consisting of five types of basis elements:

$$
\begin{array}{cl}
h_{j}, e_{j}, f_{j}, & 1 \leqq j \leqq r=\operatorname{rankg}, \\
e_{J}^{\prime}, f_{J}^{\prime}, & 1 \leqq J \leqq \frac{1}{2}(\operatorname{dimg}-3 r) .
\end{array}
$$

Here the $h_{j}$ span a Cartan subalgebra of $\mathfrak{g}$, the $e_{j}$ are root vectors of a system of simple positive roots $\alpha_{j}$, the $e_{J}^{\prime}$ are root vectors of the remaining positive roots, the $f_{j}$ and $f_{J}^{\prime}$ are root vectors of the corresponding negative roots, and $\left[e_{j}, f_{j}\right]=h_{j}$ for all $j$.

In local coordinates $\left(x^{\mu}\right)$ on $\mathbb{R}^{4}$ with the standard Riemannian metric, orientation form $d x^{0} \wedge d x^{1} \wedge d x^{2} \wedge d x^{3}$, and corresponding Hodge star operator, $* \mathrm{ad}_{F}$ maps any $\mathrm{g}$-valued 1 -form $B=B_{\sigma} d x^{\sigma}$ to the 1 -form

$$
\begin{aligned}
*[F \wedge B] & =*\left(\frac{1}{2}\left[F_{\mu v}, B_{\sigma}\right] d x^{\mu} \wedge d x^{v} \wedge d x^{\sigma}\right)=\frac{1}{2}\left[F_{\mu v}, B_{\sigma}\right] \varepsilon_{\mu v \sigma \tau} d x^{\tau} \\
& =\left[(* F)_{\sigma \tau}, B_{\sigma}\right] d x^{\tau}=\operatorname{ad}\left((* F)_{\sigma \tau}\right)\left(B_{\sigma}\right) d x^{\tau}
\end{aligned}
$$


[using ad in its standard sense $\operatorname{ad}(X)(Y)=[X, Y]]$. Hence $* \operatorname{ad}_{F}$ can be represented at $x_{0}$ by a $4 \times 4$ matrix whose $(\tau, \sigma)$ entry is the $\operatorname{dim} G \times \operatorname{dim} G$ matrix $\operatorname{ad}\left((* F)_{\sigma \tau}\right)$. Its form is

$$
\left(\begin{array}{cccc}
0 & Y_{1} & Y_{2} & Y_{3} \\
-Y_{1} & 0 & Y_{4} & Y_{5} \\
-Y_{2} & -Y_{4} & 0 & Y_{6} \\
-Y_{3} & -Y_{5} & -Y_{6} & 0
\end{array}\right) \quad Y_{i}=\operatorname{ad}\left(y_{i}\right), \quad y_{i} \in \mathfrak{g} .
$$

Since there are no a priori restrictions on the $y_{i}$, it would suffice to find a nonsingular matrix of this form having $Y_{1}=Y_{6}=0$. Hence it suffices to find $y_{2}, y_{3}, y_{4}, y_{5} \in \mathfrak{g}$ for which the determinant of the matrix

$$
C=\left(\begin{array}{ll}
Y_{2} & Y_{3} \\
Y_{4} & Y_{5}
\end{array}\right)
$$

is non-zero.

Choose $y_{2}=y_{5}$ to be any element of the Cartan subalgebra not in the kernel of any root. Thus in block form relative to the basis $\left\{h_{j}, e_{j}, f_{j}, e_{J}^{\prime}, f_{J}^{\prime}\right\}, Y_{2}=Y_{5}$ has the matrix

$$
\left(\begin{array}{lllll}
0 & & & & \\
& d & & & \\
& & -d & & \\
& & & D & \\
& & & & -D
\end{array}\right)
$$

where $d$ and $D$ are non-singular diagonal matrices. Set $y_{3}=t \sum e_{i}$ and $y_{4}=t \sum f_{i}$, where $t$ is a complex scalar parameter. Decomposing $Y_{3}$ and $Y_{4}$ into block form as was done for $Y_{2}=Y_{5}$, we find that

$$
C=\left(\begin{array}{ccccc|ccccc}
0 & & & & & 0 & 0 & t I & 0 & 0 \\
& d & & & & -t a & 0 & 0 & 0 & 0 \\
& & -d & & & 0 & 0 & 0 & 0 & t c_{1} \\
& & & D & & 0 & t c_{2} & 0 & t c_{3} & 0 \\
& & & & -D & 0 & 0 & 0 & 0 & t c_{4} \\
\hline 0 & -t I & 0 & 0 & 0 & 0 & & & & \\
0 & 0 & 0 & t c_{5} & 0 & & d & & & \\
t a & 0 & 0 & 0 & 0 & & & -d & & \\
0 & 0 & 0 & t c_{6} & 0 & & & & D & \\
0 & 0 & t c_{7} & 0 & t c_{8} & & & & & -D
\end{array}\right)
$$

Here $I$ is the $r \times r$ identity matrix, $c_{1}, c_{2}$, etc. stand for various matrices which will not concern us, and $a$ is a certain $r \times r$ matrix which is nonsingular, as we shall now show. The $(j, k)$ entry of $a$ is $\alpha_{j}\left(h_{k}\right)$, because $\left[e_{j}, h_{k}\right]=-\alpha_{j}\left(h_{k}\right) e_{j}$ and $\left[f_{j}, h_{k}\right]=\alpha_{j}\left(h_{k}\right) f_{j}$. 
Since the $h_{k}$ are a basis of the Cartan subalgebra and the simple roots $\alpha_{j}$ are a basis of its dual, no non-trivial linear combination of the rows of $a$ can be nonsingular.

We can expand $\operatorname{det} C$ by minors four times, using the lone non-zero blocks in rows 1 and 6 and those in Columns 1 and 6 . We get

$$
\operatorname{det} C= \pm t^{4 r}(\operatorname{det} a)^{2} \operatorname{det} C^{\prime}
$$

where

$$
C^{\prime}=\left(\begin{array}{cccccc}
-d & 0 & 0 & 0 & 0 & t c_{1} \\
0 & D & 0 & t c_{2} & t c_{3} & 0 \\
0 & 0 & -D & 0 & 0 & t c_{4} \\
0 & t c_{5} & 0 & d & 0 & 0 \\
0 & t c_{6} & 0 & 0 & D & 0 \\
t c_{7} & 0 & t c_{8} & 0 & 0 & -D
\end{array}\right)
$$

Now $\operatorname{det} C^{\prime}$ is a polynomial in $t$ which is non-zero when $t=0$. Hence for $t$ close to but not equal to zero, $\operatorname{det} C^{\prime}$ and $\operatorname{det} C$ are non-zero. QED

\section{Proof of Theorem 2 (Discontinuity)}

Before proving Theorem 2 we discuss the behavior of $\operatorname{ad}_{F}^{-1}(d F)$ near the zeros of det $* \mathrm{ad}_{F}$. Our calculations will be in local coordinates $x=\left(x_{0}, x_{1}, x_{2}, x_{3}\right)$ on one coordinate patch, and the notation will otherwise be the same as in Sect. 2. Once we have demonstrated that pathology exists locally we will show that it exists globally as well.

First consider the following problem. Suppose that $F$ is an arbitrary smooth $(\operatorname{ad} P)$-valued 2-form on $M$, not necessarily a curvature, and that $\mathrm{ad}_{F}$ is invertible on an open dense subset $U$ of $M$. If we assume that $F$ is actually a curvature on $U$, to wit, the curvature of the connection $A=\operatorname{ad}_{F}^{-1}(d F)$ defined on $U$, does it follow that $A$ extends smoothly to $M$ ? Such a removable singularity theorem would shed some light on the behavior of $\left(\operatorname{ad}_{F}\right)^{-1}(d F)$ at the boundary of $U$. Unfortunately, there is no such result, and the counterexample is a model for the counterexample to the continuity of the inverse of the curvature operator.

Our gauge group will be $\mathrm{SU}(2)$ [or $\mathrm{SO}(3)$ ], with Lie algebra su(2) spanned by $\left\{t_{1}, t_{2}, t_{3}\right\}$ satisfying $\left[t_{i}, t_{j}\right]=\varepsilon_{i j k} t_{k}$. Let $T_{i}$ be the matrix of ad $t_{i}$; thus

$$
T_{1}=\left(\begin{array}{rrr}
0 & 0 & 0 \\
0 & 0 & -1 \\
0 & 1 & 0
\end{array}\right) \quad T_{2}=\left(\begin{array}{rrr}
0 & 0 & 1 \\
0 & 0 & 0 \\
-1 & 0 & 0
\end{array}\right) \quad T_{3}=\left(\begin{array}{rrr}
0 & -1 & 0 \\
1 & 0 & 0 \\
0 & 0 & 0
\end{array}\right)
$$

Now let

$$
\begin{aligned}
F= & t_{1}\left(-x_{0} x_{1} d x_{2} \wedge d x_{3}+d x_{0} \wedge d x_{1}+d x_{0} \wedge d x_{2}\right) \\
& +t_{2}\left(-x_{0}^{2} d x_{1} \wedge d x_{2}+d x_{1} \wedge d x_{3}\right) \\
& +t_{3}\left(d x_{0} \wedge d x_{2}+x_{0} x_{1} d x_{1} \wedge d x_{3}-d x_{0} \wedge d x_{1}\right)
\end{aligned}
$$


so that

$$
\begin{aligned}
* F= & t_{1}\left(-x_{0} x_{1} d x_{0} \wedge d x_{1}+d x_{2} \wedge d x_{3}-d x_{1} \wedge d x_{3}\right) \\
& +t_{2}\left(-x_{0}^{2} d x_{0} \wedge d x_{3}-d x_{0} \wedge d x_{2}\right) \\
& +t_{3}\left(-d x_{1} \wedge d x_{3}-x_{0} x_{1} d x_{0} \wedge d x_{2}-d x_{2} \wedge d x_{3}\right)
\end{aligned}
$$

By Sect. 2, the matrix representing ${ }^{*} \operatorname{ad}_{F}$ is $M_{\tau \sigma}=\left(\operatorname{ad}\left(\left({ }^{*} F\right)_{\sigma \tau}\right)\right)$, which equals

$$
\left(\begin{array}{cccc}
0 & x_{0} x_{1} T_{1} & \left(T_{2}+x_{0} x_{1} T_{3}\right) & x_{0}^{2} T_{2} \\
-x_{0} x_{1} T_{1} & 0 & 0 & \left(T_{1}+T_{3}\right) \\
\left(-T_{2}-x_{0} x_{1} T_{3}\right) & 0 & 0 & \left(T_{3}-T_{1}\right) \\
-x_{0}^{2} T_{2} & \left(-T_{1}-T_{3}\right) & \left(T_{1}-T_{3}\right) & 0
\end{array}\right)
$$

The determinant of this matrix is the square of the $6 \times 6$ determinant

$$
\operatorname{det}\left(\begin{array}{cc}
x_{0} x_{1} T_{1} & \left(T_{2}+x_{0} x_{1} T_{3}\right) \\
\left(-T_{1}-T_{3}\right) & \left(T_{1}-T_{3}\right)
\end{array}\right)=-2 x_{0} x_{1} .
$$

Thus det $* \operatorname{ad}_{F}=\left(2 x_{0} x_{1}\right)^{2}$. On the set $\left\{x_{0} x_{1} \neq 0\right\}$ there is only one connection $A$ satisfying $A=\operatorname{ad}_{F}^{-1}(d F)$, namely

$$
A=t_{1} x_{0} d x_{1}+t_{2} x_{1} d x_{3}+t_{3} x_{0} d x_{2}+t_{2} d x_{0} / x_{0} .
$$

Moreover, $F$ is the curvature of $A$ on $\left\{x_{0} x_{1} \neq 0\right\}$ (in fact, on $\left\{x_{0} \neq 0\right\}$ ), though we emphasize that this was not forced by the relation $A=\operatorname{ad}_{F}^{-1}(d F)$. On the other hand, $A$ does not extend smoothly across $\left\{x_{0}=0\right\}$.

Remark 3.1. We have here an example in a non-abelian gauge theory of a singular potential whose field is nonsingular. It seems to us that this may be interesting in its own right.

Remark 3.2. For the nonsingular potential $A=t_{1} x_{0} d x_{1}+t_{2} x_{1} d x_{3}+t_{3} x_{0} d x_{2}$ we compute det $* \operatorname{ad}_{F}=\left(x_{0} x_{1}\right)^{2}$, a constant multiple of det $* \operatorname{ad}_{F}$ in the previous example of singular $A$ with nonsingular $F$. This indicates that the function det $* \mathrm{ad}_{F}$ alone does not contain enough information to determine whether $\operatorname{ad}_{F}^{-1}(d F)$ is a nonsingular potential with curvature $F$.

Proof of Theorem 2. Our proof that the curvature operator $\mathscr{F}$, restricted to the domain $\mathscr{A}^{*}$ on which it is one to one, does not have a continuous inverse is based on a counterexample much like the one above. Let

$$
A=t_{1} e^{x_{0}} f d x_{1}+t_{2} f d x_{3}+t_{3} e^{x_{0}} d x_{2},
$$

where $f$ is a function of $x_{1}$ and $x_{3}$ to be specified later. Then

$$
\begin{aligned}
* F= & -t_{1} e^{x_{0}} f d x_{0} \wedge d x_{1} \\
& +\left(t_{1} e^{x_{0}} \partial f / \partial x_{3}-t_{2} \partial f / \partial x_{1}-t_{3} e^{x_{0}} f^{2}\right) d x_{0} \wedge d x_{2} \\
& -t_{2} e^{2 x_{0}} f d x_{0} \wedge d x_{3}-t_{3} e^{x_{0}} d x_{1} \wedge d x_{3} \\
& +t_{1} e^{x_{0}} f d x_{2} \wedge d x_{3} .
\end{aligned}
$$


The matrix of $* \mathrm{ad}_{F}$ is of the form

$$
\left(\begin{array}{rrrr}
0 & -a & -b & c \\
a & 0 & 0 & d \\
b & 0 & 0 & e \\
-c & -d & -e & 0
\end{array}\right)
$$

where

$$
\left(\begin{array}{ll}
a & b \\
d & e
\end{array}\right)=\left(\begin{array}{cc}
-e^{x_{0}} f T_{1} & \left(e^{x_{0}}\left(\partial f / \partial x_{3}\right) T_{1}-\left(\partial f / \partial x_{1}\right) T_{2}-e^{x_{0}} f^{2} T_{3}\right) \\
e^{x_{0}} T_{3} & -e^{x_{0}} f T_{1}
\end{array}\right) .
$$

The determinant of this last matrix is $\left(e^{2 x_{0}} f \partial f^{\prime} / \partial x_{1}\right)^{2}$. Therefore, det*ad ${ }_{F}$ $=\left(e^{2 x_{0}} f \cdot \partial f / \partial x_{1}\right)^{4}$.

Suppose we can find smooth functions $f$ and $g_{n}(n=1,2,3, \ldots)$ of $x_{1}$ and $x_{3}$ alone satisfying:

Condition $C$. On any compact set in $\mathbb{R}^{2}$, the functions $f g_{n}$, $f \partial g_{n} / \partial x_{1}$ and $f \partial g_{n} / \partial x_{3}$, together with their partial derivatives of all orders, converge uniformly to zero. On any compact neighborhood of $(0,0)$, however, either $\partial g_{n} / \partial x_{1}$ or $\partial g_{n} / \partial x_{3}$ does not converge uniformly to zero.

Let $A_{n}$ be the connection $A+t_{3} d g_{n}$. Its curvature is

$$
F_{n}=F-\left(t_{1} f \partial g_{n} / \partial x_{1}+t_{2} e^{x_{0}} f \partial g_{n} / \partial x_{3}\right) d x_{1} \wedge d x_{3} .
$$

On any compact neighborhood of 0 in $\mathbb{R}^{4}, A_{n}-A$ does not converge uniformly to zero, while $F_{n}-F$ and all its partial derivatives do. This will give us a "local counterexample" to the continuity of $\left(\mathscr{F} \mid \mathscr{A}^{*}\right)^{-1}$ provided we can show that $A$ and $A_{n}$ are in $\mathscr{A}^{*}$. In fact, we will show that $A$ and $A_{n} \in \mathscr{A}_{28} \subset \mathscr{A}_{\infty} \subset \mathscr{A}^{*}$, showing that $\left(\mathscr{F} \mid \mathscr{A}_{k}\right)^{-1}$ is discontinuous at least for $k \geqq 28$.

Let $w \in C^{\infty}(\mathbb{R})$ satisfy $0 \leqq w \leqq 1, w(t)=0$ when $t \leqq \frac{1}{2}$ and $w(t)=1$ when $t \geqq 1$. Choose

$$
\begin{aligned}
& f\left(x_{1}, x_{3}\right)=\left(x_{1}^{2}+e^{-1 / x_{3}^{2}}\right)^{2}, \\
& g_{n}\left(x_{1}, x_{3}\right)=\frac{e^{-n^{2} / 2} w\left(n x_{3}\right)}{x_{1}^{2}+e^{-1 / x_{3}^{2}}} .
\end{aligned}
$$

We will show in Appendix 2 that this choice of $f$ and $g_{n}$ satisfies Condition $C$. Now

$$
\operatorname{det} * \operatorname{ad}_{F_{n}}=\left(e^{2 x_{0}} f \cdot\left(\partial f / \partial x_{1}-e^{x_{0}} f \partial g_{n} / \partial x_{3}\right)\right)^{4} \text {. }
$$

Since $f \partial g_{n} / \partial x_{3}$ is a polynomial of degree 2 in $x_{1}$ (the coefficients being smooth functions of $x_{3}$ ), we can regard det $*_{F_{n}}$ as a polynomial in $x_{1}$ with leading term $\left(e^{2 x_{0}} \cdot x_{1}^{4} \cdot 4 x_{1}^{3}\right)^{4}=256 e^{8 x_{0}} x_{1}^{28}$. Hence

$$
\left(\partial / \partial x_{1}\right)^{28} \operatorname{det} * \operatorname{ad}_{F_{n}}=256 \cdot 28 ! e^{8 x_{0}} \neq 0 .
$$

This proves that $A_{n} \in \mathscr{A}_{28}$; an identical calculation shows that $A \in \mathscr{A}_{28}$.

We can extend the preceding local construction to a global one on an arbitrary principal $G$-bundle $P \rightarrow M(G=\mathrm{SU}(2)$ or $\mathrm{SO}(3)$ and $\operatorname{dim} M=4)$ as follows (see 
Appendix 3 for details). First embed $\mathbb{R}^{4}$ in $M$ and extend the locally defined connection $A$ to a global one $A^{\prime}$ using a partition of unity. Then use a standard result in transversality theory to perturb $A^{\prime}$ to a connection $A^{\prime \prime}$ which is transversal to (that is, misses) $S_{28}$ and equals $A$ on some ball containing $0 \in \mathbb{R}^{4}$. Let $\varphi: M \rightarrow \mathbb{R}$ be a smooth function supported in this ball and equal to 1 on a smaller closed ball containing 0 . Define a sequence of connections $A_{n}^{\prime \prime}=A^{\prime \prime}+t_{3} d\left(\varphi g_{n}\right)$. Then $A_{n}^{\prime \prime}$ is eventually in $\mathscr{A}_{28}$ and the curvatures $F_{n}^{\prime \prime}$ converges to $F^{\prime \prime}$, but $A_{n}^{\prime \prime}$ does not converge to $A^{\prime \prime}$. This example shows that $\left(\mathscr{F} \mid \mathscr{A}_{28}\right)^{-1}$ is discontinuous. QED

\section{Appendix 1 (Supplement to proof of Lemma 1 of Sect. 2)}

The unproven claim in the proof of Lemma 1 is an application of the following general result:

Lemma A. Let $h: \mathbb{R}^{k} \times \mathbb{R}^{n} \rightarrow \mathbb{C}$ (or $\mathbb{R}$ ) be a polynomial $h(x, y)\left(x \in \mathbb{R}^{k} ; y \in \mathbb{R}^{n}\right)$ of the form

$$
h(x, y)=f(y)+g(x, y),
$$

where $f$ is a homogeneous polynomial of degree $N \geqq 1$ in (the coordinates of) $y$ alone, not identically zero, and $g$ is a polynomial in $x$ and $y$ of degree $\leqq N-1$ in $y$. Then $h^{-1}(0)$ can be decomposed as a finite union of connected analytic submanifolds $M$, with the property that every $M_{\alpha}$ has at every point a normal vector (in the standard Euclidean metric) with a non-zero component in some $y_{j}$ direction.

Proof. For each fixed $x \in \mathbb{R}^{k}, h(x, y)$ is not the zero polynomial in $y$. Hence $h^{-1}(0)$ contains no non-empty open subsets of $\{x\} \times \mathbb{R}^{n} \simeq \mathbb{R}^{n}$.

Using constructions of Whitney on algebraic sets [Mil, p. 15, proof of Corollary 2.6] decompose $h^{-1}(0)$ into a finite union of connected analytic submanifolds $L_{\beta}$, each one the difference of two algebraic sets. It follows easily, from the construction of the $L_{\beta}$ and from a theorem of Whitney [Mil, p. 11, Theorem 2.3], that for each fixed $\beta$ and each point $p \in L_{\beta}$ there exist polynomials $h_{1}, \ldots, h_{r}$, and there exists a neighborhood $U$ of $p$ such that (i) the $h_{i}$ vanish on $U \cap L_{\beta}$, and (ii) $\left\{d h_{1}, \ldots, d h_{r}\right\}$ is a basis of the covectors $\left[\right.$ in $\left.T_{(x, y)}^{*}\left(\mathbb{R}^{k} \times \mathbb{R}^{n}\right)\right]$ annihilating $T L_{\beta}$, at every point $(x, y) \in U \cap L_{\beta}$. The condition

Condition $(*)$. No normal vector of $L$ at $(x, y)$ has a component in any $y_{j}$ direction.

specialized to $L=L_{\beta}$, is an algebraic condition on the points $(x, y)$ of $L_{\beta}$, equivalent at $(x, y) \in U \cap L_{\beta}$ to the polynomial condition " $\partial h_{i} / \partial y_{j}=0$ for all $i, j$."

Consider the sequence

$$
L_{\beta}=L_{\beta}^{0} \supset L_{\beta}^{1} \supset L_{\beta}^{2} \supset \ldots,
$$

where $L_{\beta}^{m+1}$ is the algebraic subset of $L_{\beta}^{m}$ consisting of all points $(x, y) \in L_{\beta}^{m}$ satisfying Condition $(*)$ with $L=L_{\beta}^{m}$. We can decompose each $L_{\beta}^{m}-L_{\beta}^{m+1}$ into a finite union of connected analytic manifolds (by [Mil, p. 15, Corollary 2.6]), and by construction each of these has at every point a normal vector with a component in some $y_{j}$ direction. We can take these manifolds (for all $m$ and all $\beta$ ) as the $M_{\alpha}$ 
which we were to find provided that for each $\beta$, the sequence of $L_{\beta}^{m}$ ends with the empty set. Indeed, in the contrary case the sequence would stabilize at some manifold $L$ satisfying Condition $(*)$ at every point. This would imply that at every $(x, y) \in L$, the tangent space $T L$ contains every vector $\partial / \partial y_{j}$, that is, that $T\left(\{x\} \times \mathbb{R}^{n}\right)_{(x, y)} \subset T L_{(x, y)}$. But then

$$
\left\{T\left(\{x\} \times \mathbb{R}^{n}\right)_{(x, y)} \mid(x, y) \in L\right\}
$$

would be a distribution on $L$ which satisfies the Frobenius condition for integrability [KN, p. 10], since the restrictions of any two of the vector fields $\partial / \partial y_{j}$ to $L$ would have bracket product equal to zero. The leaves of the distribution would be $n$-dimensional submanifolds of $L$ containing non-empty open subsets of $\{x\} \times \mathbb{R}^{n}$, in contradiction to the fact that $h^{-1}(0)$ contains no such subsets. QED

Remark. For the proof of Lemma 1 we use a slight extension of Lemma A. Keep the notation of Lemma A. Letting $\pi: \mathbb{R}^{k} \times \mathbb{R}^{n} \times \mathbb{R}^{q} \rightarrow \mathbb{R}^{k} \times \mathbb{R}^{n}$ be the canonical projection, we decompose $(h \circ \pi)^{-1}(0)$ as $\bigcup_{\alpha} h^{-1} M_{\alpha}=\bigcup_{\alpha} M_{\alpha} \times \mathbb{R}^{q}$, and observe that at every point, $M_{\alpha} \times \mathbb{R}^{q}$ has a normal vector with a component in an $\mathbb{R}^{n}$ direction but none in any $\mathbb{R}^{q}$ direction.

Appendix 2. Proof that $f$ and $g_{n}$ satisfy Condition $C$ in the proof of Theorem 2 (Sect. 3).

We must prove that $f g_{n}, f d g_{n}$ and all their partial derivatives converge uniformly to zero on compact sets in $\mathbb{R}^{2}$ as $n \rightarrow \infty$, but that $d g_{n}$ does not converge uniformly to zero on any compact neighborhood of $(0,0)$. We compute:

$$
\begin{gathered}
d g_{n}=e^{-n^{2} / 2}\left(x_{1}^{2}+e^{-1 / x_{3}^{2}}\right)^{-2} Q_{n}, \\
f d g_{n}=e^{-n^{2} / 2} Q_{n}, \\
f g_{n}=\left(x_{1}^{2}+e^{-1 / x_{3}^{2}}\right) e^{-n^{2} / 2} w\left(x_{3}\right),
\end{gathered}
$$

where

$$
Q_{n}\left(x_{1}, x_{3}\right)=n\left(x_{1}^{2}+e^{-1 / x_{3}^{2}}\right) w^{\prime}\left(n x_{3}\right) d x_{3}-2 w\left(n x_{3}\right) \cdot\left(x_{1} d x_{1}+x_{3}^{-3} e^{-1 / x_{3}^{2}} d x_{3}\right) .
$$

At $\left(x_{1}, x_{3}\right)=(0,1 / n), Q_{n}=-2 n^{3} e^{-n^{2}} d x_{3}$, so that

$$
d g_{n}=e^{-n^{2} / 2}\left(e^{-n^{2}}\right)^{-2}\left(-2 n^{3} e^{-n^{2}} d x_{3}\right)=-2 n^{3} e^{n^{2} / 2} d x_{3},
$$

which is unbounded as $n \rightarrow \infty$. Hence $d g_{n}$ does not converge uniformly to zero near $(0,0)$.

Since multiplication of a sequence of smooth functions on $\mathbb{R}^{2}$ by a fixed smooth function preserves uniform convergence of the sequence and all its derivatives on compact sets, we can prove that $f g_{n}, f d g_{n}$ and their derivatives converge uniformly to zero on compact sets by showing that the same is true for $e^{-n^{2} / 2} w\left(n x_{3}\right)$ [and hence, by taking $d / d x_{3}$, for $n e^{-n^{2} / 2} w^{\prime}\left(n x_{3}\right)$ ]. By construction, the $k^{\text {th }}$ derivative $w^{(k)}(t)$ has a global bound $B_{k}$ on $\mathbb{R}$. Hence for all $x_{3}$,

$$
\left|\left(d / d x_{3}\right)^{k}\left(e^{-n^{2} / 2} w\left(n x_{3}\right)\right)\right| \leqq n^{k} e^{-n^{2} / 2} B_{k},
$$

which for fixed $k$ approaches 0 as $n \rightarrow \infty$. This completes the proof. 
Appendix 3. Extension from a local to a global example in the proof of Theorem 2 (Sect. 3).

Let $M$ be any 4-manifold and $P \rightarrow M$ any principal $G$-bundle over $M(G=\mathrm{SU}(2)$ or SO(3)). Let $f, g_{n}, A_{n}, A, F_{n}$, and $F$ be as in the proof of Theorem 2. Consider them to be defined on an open set $B \subset M$ which is diffeomorphic to $\mathbb{R}^{4}$ in some given way. Let $B_{r}$ be the open ball $\|x\|<r$ in $\mathbb{R}^{4} \simeq B$. Let $\mathscr{A}_{k}^{B}$, respectively $\mathscr{A}_{k}^{M}$, denote $\mathscr{A}_{k}$ for $B$, respectively for $M$, and similarly for $E^{B}, E^{M}$, etc.

Step 1. Construct (by a partition of unity) a connection $A^{\prime}$ on $M$ which equals $A$ on $B_{8}$.

Step 2. Perturb $A^{\prime}$ to get a connection $A^{\prime \prime}$ in $\mathscr{A}_{28}^{M}$ which equals $A$ on $B_{5}$. Rigorously, we choose a metric $d$ on $J^{29}\left(E^{M}\right)$ and observe, as in the proof of Lemma 2, Sect. 2, that there is a continuous function $\delta: B_{8} \rightarrow \mathbb{R}^{+}$satisfying $d\left(j^{29} A^{\prime}(x), S_{28}\right) \geqq \delta(x)$ for all $x \in B_{8}$ (with $S_{28}$ as in Sect. 2). Construct a function $\delta^{\prime}: M \rightarrow \mathbb{R}^{+}$agreeing with $\delta$ on $B_{7}$. Let

$$
V=\left\{A^{*} \in \mathscr{A}^{M} \mid d\left(j^{29} A^{\prime}(x), j^{29} A^{*}(x)\right)<\delta^{\prime}(x) \text { for all } x \in M\right\} .
$$

Then $V$ is an open neighborhood of $A^{\prime}$ in $\mathscr{A}^{M}$, and for every $A^{*} \in V, j^{29} A^{*}$ misses $S_{28}$ on $B_{7}$.

Now Corollary 4.11 of $[\mathrm{GG}, \mathrm{p} .56]$ states that if $C^{\infty}(X, Y)$ is the space of $C^{\infty}$ maps from a manifold $X$ to a manifold $Y$, endowed with the Whitney $C^{\infty}$ topology, $J^{k}(X, Y)$ is the manifold of $k$-jets of such maps, $U$ is open in $X, W$ is a submanifold of $J^{k}(X, Y)$ whose projection into $X$ has closure contained in $U, f$ is in $C^{\infty}(X, Y)$, and $V$ is a neighborhood of $f$ in $C^{\infty}(X, Y)$, then there exists a map $g \in V$ with $j^{k} g$ transversal to $W$ and $g=f$ off $U$. This result and its proof can easily be modified for spaces of sections instead of $C^{\infty}(X, Y)$. Let us apply the modified result to our problem, using $\mathscr{A}^{M}=\Gamma E^{M}$ instead of $C^{\infty}(X, Y), J^{29}(E)$ for $J^{k}(X, Y), S_{28}^{M-\bar{B}_{6}}$ for $W$, $M-\bar{B}_{5}$ for $U, A^{\prime}$ for $f, V$ as above, and $A^{\prime \prime}$ for $g$. We conclude that there exists an $A^{\prime \prime}$ satisfying $A^{\prime \prime}=A^{\prime}=A$ on $B_{5}$ and $A^{\prime \prime} \in \mathscr{A}_{28}^{M}$ (because $j^{29} A^{\prime \prime}$ is transversal to $S_{28}$ on $M-\bar{B}_{6}$ by the Corollary and on $B_{7}$ since $\left.A^{\prime \prime} \in V\right)$.

Step 3. Pick a smooth function $\varphi$ on $M$, equal to 1 on $B_{3}$ and to 0 outside $B_{4}$. Let $A_{n}^{\prime \prime}=A^{\prime \prime}+t_{3} d\left(\varphi g_{n}\right)$ (on $M$ ). Thus $A_{n}^{\prime \prime}=A^{\prime \prime}$ outside $B_{4}$ and $A_{n}^{\prime \prime}=A_{n}$ on $B_{3}$. In particular, $A_{n}^{\prime \prime}$ does not converge to $A^{\prime \prime}$ in $\mathscr{A}^{M}$. Taking curvatures we get $F_{n}^{\prime \prime}=F^{\prime \prime}$ outside $B_{4}$, while on $B_{5}$ we have

$$
F_{n}^{\prime \prime}=F^{\prime \prime}+\left(t_{1} d x_{3}-t_{2} e^{x_{0}} d x_{1}\right) f \wedge d\left(\varphi g_{n}\right)
$$

Now $f d\left(\varphi g_{n}\right)=\varphi \cdot\left(f d g_{n}\right)+\left(f g_{n}\right) d \varphi$.

Applying Condiction $C$, we see that $F_{n}^{\prime \prime}-F^{\prime \prime}$ and its derivatives converge uniformly to zero on $\bar{B}_{5}$. Since $F_{n}^{\prime \prime}=F^{\prime \prime}$ off $\bar{B}_{5}, F_{n}$ converges to $F^{\prime \prime}$ on $M$ in the Whitney $C^{\infty}$ topology (see Remark 1.1 in Sect. 1). This implies, in particular, that det ${ }^{*} \operatorname{ad}_{F_{n}^{\prime \prime}} \rightarrow \operatorname{det} * \mathrm{ad}_{F^{\prime \prime}}$ in $C^{\infty}(M)$. The set $\left\{h \in C^{\infty}(M) j^{28} h(x)\right.$ is never 0$\}$ is open in $C^{\infty}(M)$ and contains det ${ }^{*} \mathrm{ad}_{F^{\prime \prime}}$; therefore it contains det ${ }^{*} \operatorname{ad}_{F_{n}^{\prime \prime}}$ for $n$ large enough. Thus $A_{n}^{\prime \prime}$ is eventually in $\mathscr{A}_{28}$. This completes the proof that $\left(\mathscr{\mathscr { F }} \mid \mathscr{A}_{28}^{M}\right)^{-1}$ is discontinuous. QED 
Acknowledgements. This research was supported in part by N.S.F. Grant MCS 80-03419 (first author) and by an NSERCC operating grant (second author). The authors wish to thank their home institutions for supporting them during their leaves of absence in Israel for the 1981-1982 academic year, and their host institutions, Tel-Aviv University and Weizmann Institute of Science, respectively, for their hospitality and support.

\section{References}

[BK] Belifante, J.G.F., Kolman, B.: A Survey of Lie groups and Lie algebras. Philadelphia: SIAM 1972

[C] Calvo, M.: Connection between Yang-Mills potentials and their field strengths. Phys. Rev. D 15, 1733-1735 (1977)

[DX] Dao-xing, X.: On field strengths and gauge potentials of Yang-Mills' fields. Scientia Sinica 20, 145-157 (1977)

[DD] Deser, S., Drechsler, W.: Generalized gauge field copies. Phys. Lett. 86B, 189-192 (1979)

[DT] Deser, S., Teitelboim, C.: Duality transforms of abelian and non-abelian gauge fields. Phys. Rev. D 13, 1592-1597 (1976)

[DW] Deser, S., Wilczek, F.: Non-uniqueness of gauge field potentials. Phys. Lett. 65B, 391-393 (1976)

[D1] Doria, F.A.: The geometry of gauge field copies. Commun. Math. Phys. 79, 435-456 (1981)

[D2] Doria, F.A.: Quasi-abelian and fully non-abelian gauge field copies: a classification. J. Math. Phys. 22, 2943-2951 (1981)

[Fl] Flanders, H.: Differential forms with applications to the physıcal sciences. New York: Academic Press 1963

[GG] Golubitsky, M., Guillemin, V.: Stable mappings and their singularities. New York: Springer 1973

[Gr] Greub, W., Halperin, S., Vanstone, R.: Connections, curvature, and cohomology, Vol. II. New York: Academic Press 1973

[GY] Gu, C.-H., Yang, C.-N.: Some problems on the gauge field theories, II. Sci. Sin. 20, 47-55 (1977)

[H1] Halpern, M.B.: Field strength formulation of quantum chromodynamics. Phys. Rev. D 16, 1798-1801 (1977)

[H2] Halpern, M.B.: Field strength copies and action copies in quantum chromodynamics. Nucl. Phys. B 139, 477-489 (1978)

[H3] Halpern, M.B. : Field strength and dual variable formulations of gauge theory. Phys. Rev. D 19, 517-530 (1979)

[KN] Kobayashi, S., Nomizu, K.: Foundations of differential geometry, Part 1. New York: Interscience 1963

[KC] Kugler, M., Castillejo, L.: When does the Yang-Mills field determine the potential uniquely? Unpublished notes

[Mil] Milnor, J.: Singular points of complex hypersurfaces. Princeton: Princeton Univ. Press 1968

[M] Mostow, M.A.: The field copy problem: to what extent do curvature (gauge field) and it covariant derivatives determine connection (gauge potential)? Commun. Math. Phys. 78, $137-150$ (1980)

[MS] Mostow, M.A., Shnider, S. : Counterexamples to some results on the existence of field copies. Commun. Math. Phys.

[R] Roskies, R.: Uniqueness of Yang-Mills potentials. Phys. Rev. D 15, 1731-1732 (1977)

[S] Singer, I.M. : Some remarks on the Gribov ambiguity. Commun. Math. Phys. 60, 7-12 (1978)

[So] Solomon, S.: On the field strength-potential connection in non-abelian gauge theory. Nucl. Phys. B 147, 174-188 (1979)

[W] Weiss, N.: Determination of Yang-Mills potentials from the field strengths. Phys. Rev. D 20, 2606-2609 (1979)

[WY] Wu, T.-T., Yang, C.-N.: Some remarks about unquantized non-abelian gauge fields. Phys. Rev. D 12, 3843-3844 (1975)

Communicated by A. Jaffe

Received November 29, 1982; in revised form May 10, 1983 